\title{
Novel Investigation of Multivariable Conformable Calculus for Modeling Scientific Phenomena
}

\author{
Mohammed K. A. Kaabar (D), ${ }^{1,2}$ Francisco Martínez, ${ }^{3}$ Inmaculada Martínez, ${ }^{3}$ Zailan Siri (iD, ${ }^{1}$ \\ and Silvestre Paredes ${ }^{3}$ \\ ${ }^{1}$ Institute of Mathematical Sciences, Faculty of Science, University of Malaya, Kuala Lumpur 50603, Malaysia \\ ${ }^{2}$ Gofa Camp, Near Gofa Industrial College and German Adebabay, Nifas Silk-Lafto 26649, Addis Ababa, Ethiopia \\ ${ }^{3}$ Department of Applied Mathematics and Statistics, Technological University of Cartagena, Cartagena 30203, Spain
}

Correspondence should be addressed to Mohammed K. A. Kaabar; mohammed.kaabar@wsu.edu and Zailan Siri; zailansiri@um.edu.my

Received 11 September 2021; Accepted 29 October 2021; Published 23 November 2021

Academic Editor: Antonio Di Crescenzo

Copyright (C) 2021 Mohammed K. A. Kaabar et al. This is an open access article distributed under the Creative Commons Attribution License, which permits unrestricted use, distribution, and reproduction in any medium, provided the original work is properly cited.

\begin{abstract}
New investigation on the conformable version $(\mathrm{CoV})$ of multivariable calculus is proposed. The conformable derivative $(\mathrm{CoD})$ of a real-valued function (RVF) of several variables (SVs) and all related properties are investigated. An extension to vector-valued functions (VVFs) of several real variables (SRVs) is studied in this work. The CoV of chain rule (CR) for functions of SVs is also introduced. At the end, the CoV of implicit function theorem (IFThm) for SVs is established. All results in this work can be potentially applied in studying various modeling scenarios in physical oceanography such as Stommel's box model of thermohaline circulation and other related models where all our results can provide a new analysis and computational tool to investigate these models or their modified formulations.
\end{abstract}

\section{Introduction}

Many definitions of derivative have been proposed based on two categorizations: global (nonlocal) and local types. In the first one, the nonlocal fractional derivative (FrDr) is represented via integral transformation or any other related transformations where nonlocality is seen in this type along with a memory. The Riemann-Liouville and Caputo fractional definitions are considered as the most commonly known fractional definitions. Several approximate analytical and numerical methods have been recently developed to solve nonlocal fractional and local differential equations that are encountered while modeling various scientific phenomena such as the generalized Riccati expansion for solving the nonlinear KPP equation via FrDr [1] and the trigonometric quintic B-spline method for solving the nonlinear telegraph equation constructed via $\mathrm{CoV}$ [2]. The nonlinear Klein-Fock-Gordon equation has been analytically solved via two novel techniques such as the methods of generalized Riccati expansion and generalized exponential function [3]. Fractional vector (FV) calculus has been recently introduced in [4] to represent the spherical coordinates framework using the fractional derivative and integral of Caputo and Riemann-Liouville senses, respectively. FV calculus can be used effectively in modeling processes in fractal media and other fractional dynamical systems such as hydrodynamical and electrodynamical systems [4]. Fractional calculus is a powerful tool in modeling the potential flow past a sphere of an inviscid fluid [5] which is a very important research problem because this problem particularly investigates the Laplacian in three-dimensional space (spherical coordinates) which is used in governing various physical and mechanical systems in heat conduction, elasticity, Newtonian gravitational potential, ideal fluid flow, and electrostatics [6]. In addition, this problem has been applied in finding the stream function solutions for the stokes flow inside viscous sphere in an inviscid extensional flow [7]. The basics of these essential notions are mentioned in $[8,9]$. The local definition is based on certain incremental ratios. A new local derivative definition was initially 
formulated by Khalil et al. [10, 11], which is called conformable derivative (CoD). The main aim of $\mathrm{CoD}$ is to avoid some obstacles of solving nonlocal fractional differential equation where the analytical solutions can be very complicated to obtain. Consequently, several research works have mathematically analyzed some essential notions $[10,12-16]$. The Schrodinger-Hirota equation and modified KdV-Zakharov-Kuznetsov equation have been solved with the help of generalized CoDs [17]. CoDs have been employed in investigating the wick-type stochastic nonlinear evolution equations via the improved technique of Kudryashov [18] (see also the wick-type stochastic KdV equation formulated in the context of generalized CoDs [19]). According to the mathematical investigation of CoD in [20], it is clear that $\mathrm{CoD}$ cannot be addressed as a fractional derivative. Therefore, in our study, we have addressed CoD as a modified form of usual derivative which has some applications in physics and engineering due to the fact that the measurements in physics are local. Therefore, this definition is highly applicable in theoretical physics. CoD can still be helpful in many related modeling scenarios.

The CoV of analytic functions' theory has been proposed in [21]. In addition, new results are investigated on the contour conformable integral in $[22,23]$. Thus, the definition of the contour conformable integral has been utilized in [23].

Studying conformable derivative and integral is essential in various fields of natural sciences and engineering. The need for local derivative is highly appreciated in multidisciplinary sciences. While the nonlocal fractional derivatives can provide a good explanation to the dynamics of certain systems, particularly in modeling epidemic diseases, the difficulty of obtaining exact or analytical solutions to the problems formulated in the senses of nonlocal fractional derivatives can make the investigation of fractional-order systems a real challenge for researchers. Thus, researchers have paid more attention to conformable derivative and other related local derivatives in modeling scientific phenomena. While there are some recent studies concerning the mathematical analysis of conformable calculus such as the multivariable conformable calculus [15] that was introduced in 2018, the behavior of conformable derivatives of functions in arbitrary Banach spaces [24] that was investigated in 2021, the differential geometry of curves [25] that was investigated in 2019 in the senses of conformable derivatives and integrals, and the behavioral framework for the conformable linear differential systems' stability [26] that was carefully studied in 2020 to utilize the importance of CoV in modeling scenarios of control theory and power electronics, our results in this work provide a comprehensive investigation of $\alpha$-derivative of a function of SVs and all related properties, the CoV of CR for functions of SVs, and the CoV of IFThm involving many numerical examples to validate our obtained results. According to the best of our knowledge, our original investigation in this article provides an essential mathematical analysis tool for researchers working on modeling phenomena in physics and engineering in the sense of conformable calculus because all theorems and properties in this work will be needed in such modeling scenarios.
The article consists of the following sections: essential notions of the CoV of calculus are mentioned in Section 2. Then, the $\alpha$-derivative of RVF of SVs is investigated, and all its main properties are established in Section 3. Furthermore, these results are extended to the VVFs of SRVs. In addition, the CR for functions of SVs is introduced in two particular cases in Section 4. In the last part, the CoV of IFThm for SVs is obtained in Section 5 by first establishing the conformable theorem of existence and regularity of the implicit function for single equation. Second, this result is extended to a system of several equations and SRVs. Some concluding remarks are specified in Section 6.

\section{Fundamental Notions}

Definition 1 (See [10]). For a function $f:[0, \infty) \longrightarrow R$, the $\alpha^{\text {th }}$ order CoD can be written as

$$
\left(T_{\alpha} f\right)(t)=\lim _{\epsilon \longrightarrow 0} \frac{f\left(t+\varepsilon t^{1-\alpha}\right)-f(t)}{\varepsilon},
$$

$\forall t>0, \alpha \in(0,1]$. If $f$ is $\alpha$-differentiable function $(\alpha D F)$ in some $(0, a), a>0$, and $\lim _{t \longrightarrow 0^{+}}\left(T_{\alpha} f\right)(t)$ exists; then, it is expressed as

$$
\left(T_{\alpha} f\right)(0)=\lim _{t \longrightarrow 0^{+}}\left(T_{\alpha} f\right)(t)
$$

Theorem 1 (See [10]). If $f:[0, \infty) \longrightarrow R$ is $\alpha$ DF at $t_{0}>0$, $\alpha \in(0,1]$, then $f$ is continuous function $(C F)$ at $t_{0}$.

Theorem 2 (See [10]). Assuming that $\alpha \in(0,1]$ and $f, g$ are $\alpha D F s$ at a point $t>0$, we have

(i) $T_{\alpha}(a f+b g)=a\left(T_{\alpha} f\right)+b\left(T_{\alpha} g\right), \forall a, b \in R$.

(ii) $T_{\alpha}\left(t^{p}\right)=p t^{p-\alpha}, \forall p \in R$.

(iii) $T_{\alpha}(\lambda)=0, \forall$ constant functions $f(t)=\lambda$

(iv) $T_{\alpha}(f g)=f\left(T_{\alpha} g\right)+g\left(T_{\alpha} f\right)$

(v) $T_{\alpha}(f / g)=g\left(T_{\alpha} f\right)-f\left(T_{\alpha} g\right) / g^{2}$

(vi) If we suppose that $f$ is differentiable, then $\left(T_{\alpha} f\right)(t)=t^{1-\alpha} d f / d t(t)$.

From Definition 1, the CoD of some functions are expressed as

(i) $T_{\alpha}(1)=0$

(ii) $T_{\alpha}(\sin (a t))=a t^{1-\alpha} \cos (a t)$

(iii) $T_{\alpha}(\cos (a t))=-a t^{1-\alpha} \sin (a t)$

(iv) $T_{\alpha}\left(e^{a t}\right)=a t^{1-\alpha} e^{a t}, a \in R$.

Definition 2 (See [11]). The left CoD beginning from $a$ of function $f:[a, \infty) \longrightarrow R$ of order $\alpha \in(0,1]$ is expressed as

$$
\left(T_{\alpha}^{a} f\right)(t)=\lim _{\varepsilon \longrightarrow 0} \frac{f\left(t+\varepsilon(t-a)^{1-\alpha}\right)-f(t)}{\varepsilon}, \quad t>a .
$$

For $a=0$, it is expressed as $\left(T_{\alpha} f\right)(t)$. If $f$ is $\alpha D F$ in some $(a, b)$, then we set 


$$
\left(T_{\alpha}^{a} f\right)(a)=\lim _{t \longrightarrow a^{+}}\left(T_{\alpha}^{a} f\right)(t)
$$

Theorem 3 (See [12]). Suppose that $f, g:(a, \infty) \longrightarrow R$ are left $\alpha D F s$, where $\alpha \in(0,1]$. Let us assume that $h(t)=f(g(t)), h(t)$ is $\alpha D F \forall t \neq a$ and $g(t) \neq 0$, and we get

$$
\left(T_{\alpha}^{a} h\right)(t)=\left(T_{\alpha}^{a} f\right)(g(t)) \cdot\left(T_{\alpha}^{a} g\right)(t) \cdot(g(t))^{\alpha-1} .
$$

$$
\text { If } t=a \text {, then we obtain }
$$$$
\left(T_{\alpha}^{a} h\right)(a)=\lim _{t \longrightarrow a^{+}}\left(T_{\alpha}^{a} f\right)(g(t)) \cdot\left(T_{\alpha}^{a} g\right)(t) \cdot(g(t))^{\alpha-1} .
$$

Theorem 4 (Rolle's theorem (RT) [10]). Suppose that $a>0$, $\alpha \in(0,1]$, and function $f:[a, b] \longrightarrow R$ satisfies
(i) $f$ is CF on $[a, b]$
(ii) $f$ is $\alpha$ DF on $(a, b)$
(iii) $f(a)=f(b)$

Then, $\exists c \in(a, b), \ni\left(T_{\alpha} f\right)(c)=0$.

Theorem 5 (Mean value theorem (MVT) [10]). Assume that $a>0, \alpha \in(0,1]$, and function $f:[a, b] \longrightarrow R$ satisfies

(i) $f$ is CF on $[a, b]$

(ii) $f$ is $\alpha$ DF on $(a, b)$

Then, $\exists c \in(a, b), \quad \ni$ we have

$$
\left(T_{\alpha} f\right)(c)=\frac{f(b)-f(a)}{b^{\alpha} / \alpha-a^{\alpha} / \alpha} .
$$

Theorem 6 (Modified mean value theorem (MMVT) [27]). Assume that $a>0, \quad \alpha \in(0,1]$, and function $f:[a, b] \longrightarrow R$ satisfies
(i) $f$ is CF on $[a, b]$
(ii) $f$ is $\alpha$ DF on $(a, b)$

Then, $\exists c \in(a, b), \ni$ we have

$$
\frac{\left(T_{\alpha} f\right)(c)}{c^{1-\alpha} / \alpha}=\frac{f(b)-f(a)}{(b / \alpha)-(a / \alpha)} \text {. }
$$

Theorem 7 (See [13]). Suppose that $a>0, \alpha \in(0,1]$, and function $f:[a, b] \longrightarrow R$ satisfies
(i) $f$ is CF on $[a, b]$
(ii) $f$ is a DF on $(a, b)$

Then, we get

(i) If $\left(T_{\alpha} f\right)(t)>0 \forall t \in(a, b)$, then $f$ is increasing on $[a, b]$

(ii) If $\left(T_{\alpha} f\right)(t)<0 \quad \forall t \in(a, b)$, then $f$ is decreasing on $[a, b]$

Let us express the CoV of partial derivative (PDr) of a real-valued function (RVF) with SVs as follows.

Definition 3 (See $[14,15]$ ). Assume that $f$ is a RVF with $n$ variables, and there is a point $\mathbf{a}=\left(a_{1}, \ldots, a_{n}\right) \in R^{n}$, where its $i^{\text {th }}$ component is positive. Then, the limit is written as

$$
\lim _{\varepsilon \longrightarrow 0} \frac{f\left(a_{1}, \ldots, a_{i}+\varepsilon a_{i}{ }^{1-\alpha}, \ldots, a_{n}\right)-f\left(a_{1}, \ldots, a_{n}\right)}{\varepsilon} .
$$

If the above limit exists, the $i^{\text {th }} \mathrm{CoV}$ of PDr of $f$ of the order $\alpha \in(0,1]$ at $\mathbf{a}$, represented by $\partial^{\alpha} / \partial x_{i}^{\alpha} f(\mathbf{a})$.

\section{3. $\alpha$-Derivative of a RVF of SVs}

Definition 4. Suppose that $f$ is a RVF with $n$ variables $x_{1}, \ldots, x_{n}$, and $\alpha \in(0,1]$. Then, we say that $f$ is $\alpha D F$ at $\mathbf{a}=\left(a_{1}, \ldots, a_{n}\right) \in R^{n}$, each $a_{i}>0$, if any of the three conditions which are equivalent to each other is verified:

(i) There is a linear transformation $L: R^{n} \longrightarrow R$, such that

$$
\lim _{\mathbf{h} \longrightarrow 0} \frac{f\left(a_{1}+h_{1} a_{1}^{1-\alpha}, \ldots, a_{n}+h_{n} a_{n}{ }^{1-\alpha}\right)-f\left(a_{1}, \ldots, a_{n}\right)-L(h)}{\|\mathbf{h}\|}=0,
$$

Where $\mathbf{h}=\left(h_{1}, \ldots, h_{n}\right), \quad\|\mathbf{h}\|=\sqrt{h_{1+\ldots+}^{2} h_{n}^{2}}, \quad$ and $\alpha \in(0,1]$. (ii) There is a linear transformation $L: R^{n} \longrightarrow R$ and a function $\varepsilon: \mathbf{h} \longrightarrow \varepsilon(\mathbf{h})$, such that

$$
f\left(a_{1}+h_{1} a_{1}{ }^{1-\alpha}, \ldots, a_{n}+h_{n} a_{n}{ }^{1-\alpha}\right)-f\left(a_{1}, \ldots, a_{n}\right)=L(\mathbf{h})+\boldsymbol{\varepsilon}(\mathbf{h})\|\mathbf{h}\|,
$$

And $\lim _{\mathbf{h} \longrightarrow 0} \varepsilon(\mathbf{h})=0$. 
(iii) There is a linear transformation $L: R^{n} \longrightarrow R$ and $n$ functions $\varepsilon_{i}: \mathbf{h} \longrightarrow \varepsilon_{i}(\mathbf{h}) \forall$

$$
\begin{aligned}
& i=1,2, \ldots n, \ni \\
& f\left(a_{1}+h_{1} a_{1}{ }^{1-\alpha}, \ldots, a_{n}+h_{n} a_{n}{ }^{1-\alpha}\right)-f\left(a_{1}, \ldots, a_{n}\right)=L(\mathbf{h})+\sum_{i=1}^{n} \varepsilon_{i}(\mathbf{h}) h_{i},
\end{aligned}
$$

$$
\text { And } \lim \varepsilon_{i h \longrightarrow 0}(h)=0 \text { for } i=1,2, \ldots, n \text {. }
$$

The linear transformation $L: R^{n} \longrightarrow R$ is defined by $L(\mathbf{h})=\sum_{i=1}^{n} \alpha_{i} h_{i}$, with $\mathbf{h}=\left(h_{1}, \ldots, h_{n}\right)$ and $\alpha_{1}, \ldots, \alpha_{n} \in R$. This linear transformation is denoted by $D^{\alpha} f(\mathbf{a})$, which is called CoD of $f$ of the order $\alpha \in(0,1]$ at $\mathbf{a}$.

Remark 1. The equivalence of conditions (i) and (ii) is immediate, since

$$
\lim _{\mathbf{h} \longrightarrow 0} \mathcal{E}(\mathbf{h})=0 \leftrightarrow \varepsilon(\mathbf{h})\|h\|=o(\|\mathbf{h}\|) .
$$

To see the equivalence between conditions (ii) and (iii), we take

$$
\begin{aligned}
\varepsilon_{i} & =\varepsilon(\mathbf{h}) \frac{h_{i}}{\|\mathbf{h}\|}, \underset{n}{n=1}, \varepsilon_{i}(\mathbf{h}) h_{i} . \\
\mathcal{E}(\mathbf{h}) & =\frac{1}{\|\mathbf{h}\|} \sum
\end{aligned}
$$

As $\left|h_{i} /\|\mathbf{h}\|\right| \leq 1$, then we have the following:

(i) If $\lim \varepsilon_{\mathbf{h} \longrightarrow 0}$ (h) $=0$, then $\lim \varepsilon_{i \mathbf{h} \longrightarrow 0}(\mathbf{h})=0$

(ii) If $\lim \varepsilon_{i \mathbf{h} \longrightarrow 0}(h)=0$ for $i=1, \ldots, n$, then we obtain

$$
\lim _{\mathbf{h} \longrightarrow 0}\|\boldsymbol{\varepsilon}(\mathbf{h})\| \leq \lim _{\mathbf{h} \longrightarrow 0} \frac{1}{\|\mathbf{h}\|} \sum_{i=1}^{n}\left\|\varepsilon_{i}(\mathbf{h})\right\| \leq \lim _{\mathbf{h} \longrightarrow 0} \sum_{i=1}^{n}\left\|\varepsilon_{i}(h)\right\|=0 .
$$

i.e., $\lim _{\mathbf{h} \longrightarrow 0} \varepsilon(\mathbf{h})=0$. Hence, the conditions (ii) and (iii) are equivalent.

Example 1. Consider a function $f$ defined by $f(x, y)=e^{x}$ 2 cosy and a point $(a, b) \in R^{2}$, with $a>0$ and $b>0$; then, $D^{\alpha} f(a, b)\left(h_{1}, h_{2}\right)=h_{1} a^{1-\alpha} e^{\alpha}+2 h_{2} b^{1-\alpha} \sin b$.

Solution: to prove this, let us note that

$$
\begin{aligned}
& \lim _{\left(h_{1}, h_{2}\right) \longrightarrow(0,0)} \frac{f\left(a+a^{1-\alpha} h_{1}, b+b^{1-\alpha} h_{2}\right)-f(a, b)-L\left(h_{1}, h_{2}\right)}{\left\|\left(h_{1}, h_{2}\right)\right\|} \\
& \quad=\lim _{\left(h_{1}, h_{2}\right) \longrightarrow(0,0)} \frac{e^{a+a^{1-\alpha} h_{1}}-2 \cos \left(b+b^{1-\alpha} h_{2}\right)-\left(e^{a}-2 \cos b\right)-\left(h_{1} a^{1-\alpha} e^{a}+2 h_{2} b^{1-\alpha} \sin b\right)}{\sqrt{h_{1}^{2}+h_{2}^{2}}} \\
& \leq \lim _{h_{1} \longrightarrow 0} \frac{e^{a+a^{1-\alpha} h 1}-e^{a}-h_{1} a^{1-\alpha} e^{a}}{h_{1}}-2 \lim _{h_{2} \longrightarrow 0} \frac{\cos \left(b+b^{1-\alpha} h_{2}\right)-\cos b+b^{1-\alpha} \sin b}{h_{2}} \\
& \quad=\lim _{h_{1} \longrightarrow 0}\left(\frac{e^{a+a^{1-\alpha} h 1}-e^{a}}{h_{1}}-a^{1-\alpha} e^{a}\right)-2 \lim _{h_{2} \longrightarrow 0}\left(\frac{\cos \left(b+b^{1-\alpha} h_{2}\right)-\cos b}{h_{2}}+b^{1-\alpha} \sin b\right)=\left(a^{1-\alpha} e^{a}-a^{1-\alpha} e^{a}\right) \\
& \quad-2\left(-b^{1-\alpha} \sin b+b^{1-\alpha} \sin b\right)=0 .
\end{aligned}
$$

Theorem 8. If a RVF $f$ with $n$ variables $\alpha D F$ at Proof. Since $f$ is $\alpha D F$ at $a$, we can write the following: $\mathbf{a}=\left(a_{1}, \ldots, a_{n}\right) \in R^{n}$, each $a_{i}>0$, then $f$ is $C F$ at $\mathbf{a} \in R^{n}$.

$$
f\left(a_{1}+h_{1} a_{1}{ }^{1-\alpha}, \ldots, a_{n}+h_{n} a_{n}{ }^{1-\alpha}\right)-f\left(a_{1}, \ldots, a_{n}\right)=\sum_{i=1}^{n} \alpha_{i} h_{i}+o(\|\mathbf{h}\|) .
$$


By taking the limits of the two sides of the equality as $\mathbf{h} \longrightarrow 0$, we have

$$
\lim _{h \longrightarrow 0} f\left(a_{1}+h_{1} a_{1}{ }^{1-\alpha}, \ldots, a_{n}+h_{n} a_{n}{ }^{1-\alpha}\right)=f\left(a_{1}, \ldots, a_{n}\right) \text {. }
$$

Hence, $f$ is CF at $\mathbf{a} \in R^{n}$.

Theorem 9. If a RVF $f$ with $n$ variables is $\alpha D F$ at $\mathbf{a}=\left(a_{1}, \ldots, a_{n}\right) \in R^{n}$, each $a_{i}>0$, then $\partial^{\alpha} / \partial x_{i}^{\alpha} f(\mathbf{a})$ exists for $1 \leq i \leq n$ and the CoD of $f$ of the order $\alpha \in(0,1]$ is expressed as

$$
D^{\alpha} f(\mathbf{a})(\mathbf{h})=\sum_{i=1}^{n} \frac{\partial^{\alpha}}{\partial x_{i}^{\alpha}} f(\mathbf{a}) h_{i}
$$

where $\mathbf{h}=\left(h_{1}, \ldots, h_{n}\right)$.

Proof. By setting $h_{j}=0, \forall j \neq i$ in the formula (12), we have

$$
f\left(a_{1}, . ., a_{i}+h_{i} a_{i}{ }^{1-\alpha}+\cdots+a_{n}\right)-f\left(a_{1}, \ldots, a_{n}\right)=\alpha_{i} h_{i}+\varepsilon_{i}(\mathbf{h}) h_{i} .
$$

By multiplying by $1 / h_{i}$, we can write

$$
\frac{f\left(a_{1}, . ., a_{i}+h_{i} a_{i}{ }^{1-\alpha}+\cdots+a_{n}\right)-f\left(a_{1}, \ldots, a_{n}\right)}{h_{i}}=\alpha_{i}+\varepsilon_{i}(\mathbf{h}) \text {. }
$$

By taking the limits of the two sides of the equality as $h_{i} \longrightarrow 0$, we have

$$
\alpha_{i}=\frac{\partial^{\alpha}}{\partial x_{i}^{\alpha}} f(\mathbf{a}), \quad \forall i=1,2, \ldots, n .
$$

Finally, by substituting the values above $\alpha_{i}$ in the formula $D^{\alpha} f(\mathbf{a})(\mathbf{h})=\sum_{i=1}^{n} \alpha_{i} h_{i}$, the result is followed.
Corollary 1. If a RVF $f$ with $n$ variables is $\alpha D F$ at $\mathbf{a}=\left(a_{1}, \ldots, a_{n}\right) \in R^{n}$, each $a_{i}>0$, then $D^{\alpha} f(\mathbf{a})$ is unique.

Remark 2. If a RVF $f$ with $n$ variables is $\alpha D F$ at $\mathbf{a}=\left(a_{1}, \ldots, a_{n}\right) \in R^{n}$, each $a_{i}>0$, then the CoV of gradient of $f$ of the order $\alpha \in(0,1]$ at $a$ is

$$
\nabla^{\alpha} f(\mathbf{a})=\left(\frac{\partial^{\alpha}}{\partial x_{1}^{\alpha}} f(\mathbf{a}), \ldots, \frac{\partial^{\alpha}}{\partial x_{n}^{\alpha}} f(\mathbf{a})\right)
$$

Also, the matrix form (MF) of equation (19) is given as follows:

$$
D^{\alpha} f(\mathbf{a})(\mathbf{h})=\nabla^{\alpha} f(\mathbf{a}) \cdot \mathbf{h}=\left(\frac{\partial^{\alpha}}{\partial x_{1}^{\alpha}} f(a), \ldots, \frac{\partial^{\alpha}}{\partial x_{n}^{\alpha}} f(a)\right) \cdot\left(\begin{array}{c}
h_{1} \\
\vdots \\
h_{n}
\end{array}\right) .
$$

Theorem 10. Let $\alpha \in(0,1], f, g: X \longrightarrow R$ be a $R V F$ defined in an open set (OS): $X \subset R^{n}, \ni \forall \mathbf{x}=\left(x_{1}, \ldots, x_{n}\right) \in X$, each $x_{i}>0$, and a point $\mathbf{a}=\left(a_{1}, \ldots, a_{n}\right) \in X$. If $f, g$ are $\alpha$ DF at $\mathbf{a}$, then we have

$$
\begin{aligned}
& \text { (i) } D^{\alpha}(\lambda f+\mu g)(\mathbf{a})=\lambda D^{\alpha}(f)(\mathbf{a})+\mu D^{\alpha}(g)(\mathbf{a}), \\
& \forall \lambda, \mu \in R . \\
& \text { (ii) } D^{\alpha}(f g)(\mathbf{a})=D^{\alpha}(f)(\mathbf{a}) \cdot g(\mathbf{a})+f(\mathbf{a}) D^{\alpha}(g)(\mathbf{a})
\end{aligned}
$$

Proof. (i) follows from Definition 4; thus, it follows the proof of (i).

For (ii), let $\mathbf{A}=\left(a_{1}+h_{1} a_{1}^{1-\alpha}, \ldots, a_{n}+h_{n} a_{n}^{1-\alpha}\right)$, and then, we have

$$
\begin{aligned}
& \lim _{\mathbf{h} \longrightarrow 0} \frac{((f g)(\mathbf{A})-(f g)(\mathbf{a}))-\left(D^{\alpha} f(\mathbf{a}) \cdot g(\mathbf{a})+f(\mathbf{a}) \cdot D^{\alpha} g(\mathbf{a})\right)(\mathbf{h})}{\|\mathbf{h}\|} \\
& \quad=\lim _{\mathbf{h} \longrightarrow 0}\left(\frac{(f(\mathbf{A})-f(\mathbf{a}))-D^{\alpha} f(\mathbf{a})(\mathbf{h})}{\|\mathbf{h}\|} \cdot g(\mathbf{a})+f(\mathbf{a}) \cdot \frac{(g(\mathbf{A})-g(\mathbf{a}))-D^{\alpha} g(\mathbf{a})(\mathbf{h})}{\|\mathbf{h}\|}\right)+\lim _{\mathbf{h} \longrightarrow 0} \frac{(f(\mathbf{A})-f(\mathbf{a})) \cdot(g(\mathbf{A})-g(\mathbf{a}))}{\|\mathbf{h}\|} \\
& \quad=0+0+\lim _{\mathbf{h} \longrightarrow 0} \frac{\left(D^{\alpha} f(\mathbf{a})(\mathbf{h})\right) \cdot\left(D^{\alpha} g(\mathbf{a})(\mathbf{h})\right)}{\|\mathbf{h}\|}=\lim _{\mathbf{h} \longrightarrow 0}\left(D^{\alpha} f(\mathbf{a})\left(\frac{\mathbf{h}}{\|\mathbf{h}\|}\right)\right) \cdot\left(D^{\alpha} g(\mathbf{a})\left(\frac{\mathbf{h}}{\|\mathbf{h}\|}\right)\right) \cdot\|\mathbf{h}\|=0 .
\end{aligned}
$$

Theorem 11. Let $\alpha \in(0,1], f: X \longrightarrow R$ be a $R V F$ defined in an OS: $X \subset R^{n}, \ni \forall \mathbf{x}=\left(x_{1}, \ldots, x_{n}\right) \in X$, each $x_{i}>0$, and $a$ point $\mathbf{a}=\left(a_{1}, \ldots, a_{n}\right) \in X$. If the function $f$ has all CoV of PDrs of the order $\alpha$ at each point of a neighbourhood of the point a, $U(\mathbf{a})$, with $U(\mathbf{a}) \subset X$, and they are continuous at a, then $f$ is $\alpha$ DF at a.

Proof. See Theorem 2.1 proof in [27].
Remark 3. The above theorem allows defining the space of RVFs with $n$ variables by having continuous CoV of PDrs of order $\alpha \in(0,1]$ in a domain $X \subset R^{n}$, which can be denoted by $C_{\alpha}(X, R)$.

Finally, we can easily extend all of the above results to the VVFs of SRVs.

Theorem 12. Assume that $\alpha \in(0,1], \mathbf{f}: X \longrightarrow R^{m}$ be a VVF defined in an OS: $X \subset R^{n}, \ni \forall \mathbf{x}=\left(x_{1}, \ldots, x_{n}\right) \in X$, each 
$x_{i}>0$, and the point $\mathbf{a}=\left(a_{1}, \ldots, a_{n}\right) \in X$. The function $\mathbf{f}$ is $\alpha D F$ at $\mathbf{a}$ iff its components are $\alpha D F$ at $\mathbf{a}$, and if these components are $f_{1}, f_{2}, \ldots, f_{m}$, then the components of $D^{\alpha} \mathbf{f}(\mathbf{a})$ are the $\alpha$-derivatives $D^{\alpha} f_{j}(\mathbf{a})$, for $j=1,2, \ldots, m$, i.e.,

$$
f=\left(f_{1}, f_{2}, \ldots, f_{m}\right) \Rightarrow D^{\alpha} \mathbf{f}(\mathbf{a})=\left(D^{\alpha} f_{1}(\mathbf{a}), D^{\alpha} f_{2}(\mathbf{a}), \ldots, D^{\alpha} f_{m}(\mathbf{a})\right)
$$

Proof. It is similarly proven as same as traditional calculus by applying $D^{\alpha}$ instead of derivative.

Remark 4. Assume that $\alpha \in(0,1], \mathbf{f}: X \longrightarrow R^{m}$ is a VVF defined in an OS: $X \subset R^{n}, \ni \forall \mathbf{x}=\left(x_{1}, \ldots, x_{n}\right) \in X$, each $x_{i}>0$, and a point $\mathbf{a}=\left(a_{1}, \ldots, a_{n}\right) \in X$. If function $\mathbf{f}$ is $\alpha$-differentiable at a, then $\partial^{\alpha} / \partial x_{i}^{\alpha} f_{j}(\mathbf{a})$ exists for $i=1,2, \ldots, n, j=1,2, \ldots, m$, and the CoV of $\alpha$-Jacobian of $f$ of order $\alpha \in(0,1]$ at $\mathbf{a}$ is expressed as

$$
J^{\alpha} \mathbf{f}(\mathbf{a})=\left(\begin{array}{ccc}
\frac{\partial^{\alpha}}{\partial x_{1}^{\alpha}} f_{1}(\mathbf{a}) & \ldots & \frac{\partial^{\alpha}}{\partial x_{n}^{\alpha}} f_{1}(\mathbf{a}) \\
\vdots & \ldots & \vdots \\
\frac{\partial^{\alpha}}{\partial x_{1}^{\alpha}} f_{m}(\mathbf{a}) & \ldots & \frac{\partial^{\alpha}}{\partial x_{n}^{\alpha}} f_{m}(\mathbf{a})
\end{array}\right) .
$$

\section{The Chain Rule}

Let us prove the CR for the functions of SVs in 2 particular cases. For the proof s purpose, the continuity's hypothesis of PDrs is given [28].

Theorem 13 (CR). Suppose that $t \in R$ and $\mathbf{x}=\left(x_{1}, \ldots, x_{n}\right) \in R^{n}$. If $\mathbf{f}(t)=\left(f_{1}(t), \ldots, f_{n}(t)\right)$ is $\alpha D F$ at $a>0 \quad \ni \quad \alpha \in(0,1]$ and $a$ RVF $g$, with $n$ variables $x_{1}, \ldots, x_{n}$, is $\alpha D F$ at $f(a) \in R^{n}$, all $f_{i}(a)>0 \alpha \in(0,1]$. Then, the composition $g \circ f$ is $\alpha$-differential at $a$ and

$$
\left(T_{\alpha} g \circ \mathbf{f}\right)(a)=\sum_{i=1}^{n} \frac{\partial^{\alpha}}{\partial x_{i}^{\alpha}} g(f(a)) \cdot\left(f_{i}(a)\right)^{\alpha-1} \cdot\left(T_{\alpha} f_{i}\right)(a) .
$$

Proof. Assumeg $\in C^{1}(U(\mathbf{f}(a)), R), \ni U(\mathbf{f}(a))$ is the point $f(a) \quad$ neighborhood. Suppose that $h(t)=(g \circ \mathbf{f})(t)=g(\mathbf{f}(t))$. By setting $u=a+\varepsilon a^{1-\alpha} \quad$ in Definition 1 , we see that

$$
\left(T_{\alpha} h\right)(a)=\lim _{t \longrightarrow a} \frac{h(t)-h(a)}{t-a} a^{1-\alpha}=\lim _{t \longrightarrow a} \frac{g(\mathbf{f}(t))-g(\mathbf{f}(a))}{t-a} a^{1-\alpha} .
$$

Without loss of generality (WLOG), $U(\mathbf{f}(a))$ is assumed to be an open ball (OB), represented by $B(\mathbf{f}(a), r)$. Since $f$ is a CF, then along with points $\left(f_{1}(a), \ldots, f_{n}(a)\right)$ and $\left(f_{1}(t), \ldots, f_{n}(t)\right)$, the points $\left(f_{1}(a), f_{2}(t), \ldots, f_{n}(t)\right), \ldots, \quad\left(f_{1}(a), f_{2}(a), \ldots, f_{n}(t)\right)$ and lines connecting them must also be the ball $B(f(a), r)$. In fact, using the classical MVT for differentiable functions (DFs) of one variable is in the following computation [28]:

$$
\begin{aligned}
\frac{h(t)-h(a)}{t-a} a^{1-\alpha}= & \frac{g(\mathbf{f}(t))-g(\mathbf{f}(a))}{t-a} a^{1-\alpha} \\
= & \frac{g\left(f_{1}(t), \ldots, f_{n}(t)\right)-g\left(f_{1}(a), f_{2}(t), \ldots, f_{n}(t)\right)}{t-a} a^{1-\alpha} \\
& +\frac{g\left(f_{1}(a), f_{2}(t), \ldots, f_{n}(t)\right)-g\left(f_{1}(a), f_{2}(a), f_{3}(t), \ldots, f_{n}(t)\right)}{t-a} a^{1-\alpha}+\ldots \\
& +\frac{g\left(f_{1}(a), f_{2}(a), \ldots, f_{n}(t)\right)-g\left(f_{1}(a), \ldots, f_{n}(a)\right)}{t-a} a^{1-\alpha} \\
= & \frac{\partial}{\partial x_{1}} g\left(c_{1}, f_{2}(t), \ldots, f_{n}(t)\right) \frac{f_{1}(t)-f_{1}(a)}{t-a} a^{1-\alpha} \\
= & \frac{\partial}{\partial x_{2}} g\left(f_{1}(a), c_{2}, \ldots, f_{n}(t)\right) \frac{f_{2}(t)-f_{2}(a)}{t-a} a^{1-\alpha}+\ldots \\
& +\frac{\partial}{\partial x_{2}} g\left(f_{1}(a), c_{2}, \ldots, f_{n}(t)\right) \frac{f_{2}(t)-f_{2}(a)}{t-a} a^{1-\alpha}+\ldots \\
& +\frac{\partial}{\partial x_{n}} g\left(f_{1}(a), f_{2}(a), \ldots, c_{n}\right) \frac{f_{n}(t)-f_{n}(a)}{t-a} a^{1-\alpha},
\end{aligned}
$$


By taking limits as $t \longrightarrow a$ and using the continuity of PDrs of $g$ as well as the fact that $c_{i} \longrightarrow f_{i}(a) \forall$ $i=1,2, \ldots, n$, equation (29) is expressed as

$$
\begin{aligned}
\left(T_{\alpha} h\right)(a)= & \lim _{t \longrightarrow a} \frac{h(t)-h(a)}{t-a} a^{1-\alpha}=\lim _{t \longrightarrow a} \frac{g(\mathbf{f}(t))-g(\mathbf{f}(a))}{t-a} a^{1-\alpha} \\
= & \lim _{t \longrightarrow \infty}\left(\frac{\partial}{\partial x_{1}} g\left(c_{1}, f_{2}(t)\right) \frac{f_{1}(t)-f_{1}(a)}{t-a} a^{1-\alpha}+\cdots\right) \\
& +\frac{\partial}{\partial x_{2}} g\left(f_{1}(a), c_{2}, \ldots, f_{n}(t)\right) \frac{f_{2}(t)-f_{2}(a)}{t-a} a^{1-\alpha}+\cdots \\
& \left.+\frac{\partial}{\partial x_{n}} g\left(f_{1}(a), f_{2}(a)^{1-\alpha}, \ldots, c_{n}\right) \frac{f_{n}(t)-f_{n}(a)}{t-a} a^{1-\alpha}\right)+\frac{\partial}{\partial x_{1}} g(f(a)) \cdot f_{1}^{(1)}(a) \cdot a^{1-\alpha} \\
& +\frac{\partial}{\partial x_{2}} g(f(a)) \cdot f_{2}^{(1)}(a) \cdot a^{1-\alpha} \cdot f_{1}(a)^{\alpha-1} \cdot f_{1}^{(1)}(a) \cdot a^{1-\alpha} \\
= & \frac{\partial}{\partial x_{1}} g(f(a)) \cdot f_{1}(a)^{1-\alpha} \cdot f_{1}(a)^{1-\alpha} \cdot f_{1}(a)^{\alpha-1} \cdot f_{1}^{(1)}(a) \cdot a^{1-\alpha}+\frac{\partial}{\partial x_{2}} g(f(a)) \cdot f_{2}(a)^{1-\alpha} \\
& \cdot f_{2}(a)^{\alpha-1} \cdot f_{2}^{(1)}(a) \cdot a^{1-\alpha}+\cdots+\frac{\partial}{\partial x_{n}} g(\mathbf{f}(a)) \cdot f_{2}(a)^{1-\alpha} \cdot f_{2}(a)^{\alpha-1} \cdot f_{n}^{(1)}(a) \cdot a^{1-\alpha} \\
& +\frac{\partial^{\alpha}}{\partial x_{n}^{\alpha}} g(\mathbf{f}(a)) \cdot f_{n}(a)^{\alpha-1} \cdot\left(T_{\alpha} f_{1}\right)(\alpha) \cdot \\
= & \frac{\partial^{\alpha}}{\partial x_{1}^{\alpha}} g(\mathbf{f}(a)) \cdot f_{1}(a)^{\alpha-1} \cdot\left(T_{\alpha} f_{1}\right)(\alpha)+\frac{\partial^{\alpha}}{\partial x_{2}^{\alpha}} g(\mathbf{f}(a)) \cdot f_{2}(a)^{\alpha-1} \cdot\left(T_{\alpha} f_{2}\right)(a)+\cdots \\
& \\
& \\
& \\
&
\end{aligned}
$$

Our proof is completely done.

Remark 5. The MF of equation (28) is expressed as

$$
\begin{aligned}
D^{\alpha}(g \cdot \mathbf{f})(a)(h) & =\left(\frac{\partial^{\alpha}}{\partial x_{1}^{\alpha}} g(\mathbf{f}(a)), \ldots, \frac{\partial^{\alpha}}{\partial x_{n}^{\alpha}} g(\mathbf{f}(a))\right) \\
& \cdot\left(\begin{array}{ccc}
f_{1}(a)^{\alpha-1} & 0 & 0 \\
0 & \ddots & 0 \\
0 & 0 & f_{n}(a)^{\alpha-1}
\end{array}\right) \cdot\left(\begin{array}{c}
\left(T_{\alpha} f_{1}\right)(a) \\
\vdots \\
\left(T_{\alpha} f_{n}\right)(a)
\end{array}\right) \cdot h .
\end{aligned}
$$

Theorem 14 (CR). Let $\mathbf{x}=\left(x_{1}, \ldots, x_{n}\right) \in R^{n}$ and $\mathbf{y}=\left(y_{1}, \ldots, y_{m}\right) \in R^{m}$. If $\mathbf{f}\left(x_{1}, \ldots, x_{n}\right)=\left(f_{1}\left(x_{1}, \ldots\right.\right.$, $\left.\left.x_{n}\right), \ldots, f_{m}\left(x_{1}, \ldots, x_{n}\right)\right)$ is a $\alpha D F$ at $\mathbf{a}=\left(a_{1}, \ldots, a_{n}\right) \in R^{n}$, each $a_{i}>0 \ni \alpha \in(0,1]$, and a RVF $g$ with variables $y_{1}, \ldots, y_{m}$ is $\alpha$ DF at $\mathbf{f}(\mathbf{a}) \in R^{n}$, where all $f_{i}(a)>0 \ni \alpha \in(0,1]$. Then, the composition $g \circ \mathbf{f}$ is $\alpha$-differentiable at $a$, and we have

$$
\begin{aligned}
& \frac{\partial^{\alpha}}{\partial x_{i}^{\alpha}}(g \circ \mathbf{f})(\mathbf{a})=\sum_{j=1}^{m} \frac{\partial^{\alpha}}{\partial y_{j}^{\alpha}} g(\mathbf{f}(\mathbf{a})) \cdot f_{j}(\mathbf{a})^{\alpha-1} \cdot \frac{\partial^{\alpha}}{\partial x_{i}^{\alpha}} f_{j}(\mathbf{a}) \\
& \forall i=1,2, \ldots, n .
\end{aligned}
$$


Proof. Proof. According to PDr's definition and Theorem 14 , the following is implied:
Remark 6. The MF of equation (33) is expressed as

$$
D^{\alpha}(g \circ \mathbf{f})(\mathbf{a})(\mathbf{h})=\left(\frac{\partial^{\alpha}}{\partial y_{1}^{\alpha}} g(\mathbf{f}(\mathbf{a})), \ldots, \frac{\partial^{\alpha}}{\partial y_{m}^{\alpha}} g(\mathbf{f}(\mathbf{a}))\right) \cdot\left(\begin{array}{cccc}
f_{1}(\mathbf{a})^{\alpha-1} & 0 & 0 \\
0 & \ddots & 0 \\
0 & 0 & f_{m}(\mathbf{a})^{\alpha-1}
\end{array}\right) \cdot\left(\begin{array}{ccc}
\frac{\partial^{\alpha}}{\partial x_{1}^{\alpha}} f_{1}(\mathbf{a}) & \ldots & \frac{\partial^{\alpha}}{\partial x_{n}^{\alpha}} f_{1}(\mathbf{a}) \\
\ldots & \ldots & \ldots \\
\frac{\partial^{\alpha}}{\partial x_{1}^{\alpha}} f_{n}(\mathbf{a}) & \ldots & \frac{\partial^{\alpha}}{\partial x_{n}^{\alpha}} f_{n}(\mathbf{a})
\end{array}\right) \cdot\left(\begin{array}{c}
h_{1} \\
\vdots \\
h_{n}
\end{array}\right)
$$

\section{Conformable Implicit Function Theorem}

In this section, the CoV of IFThm for SVs is obtained. First, we establish the conformable theorem of existence and regularity of the implicit function for the case of a single equation.

Theorem 15 (Conformable implicit function theorem for the case of a single equation). Suppose that $\alpha \in(0,1]$, $F: X \longrightarrow R$ is a RVF defined in an OS: $X \subset R^{n+1}, \ni \forall$ $\left(x_{1}, \ldots, x_{n}, y\right) \in X$, each $x_{i}, y>0$, and point $\left(a_{1}, \ldots, a_{n}, b\right) \in X$. Suppose that
(i) $F\left(a_{1}, \ldots, a_{n}, b\right)=0$
(ii) $F \in C_{\alpha}(X, R)$
(iii) $\partial^{\alpha} / \partial y^{\alpha} F\left(a_{1}, \ldots, a_{n}, b\right) \neq 0$

Then, there is a neighbourhood $U \subset R^{n}$ of $\left(a_{1}, \ldots, a_{n}\right)$, such that there is a unique function (UF) $y=g\left(x_{1}, \ldots, x_{n}\right)$ that satisfies the following:

$$
g\left(a_{1}, \ldots, a_{n}\right)=b \quad \text { and } \quad F\left(x_{1}, \ldots, x_{n}, g\left(x_{1}, \ldots, x_{n}\right)\right)
$$
$=0, \forall\left(x_{1}, \ldots, x_{n}\right) \in U$

Finally, $y=g\left(x_{1}, \ldots, x_{n}\right)$ is $C_{\alpha}$ in $U$, and for every $i=1,2, \ldots, n$, we have

$$
\frac{\partial^{\alpha}}{\partial x_{i}^{\alpha}} g\left(x_{1}, \ldots, x_{n}\right)=-\frac{\partial^{\alpha} / \partial x_{i}^{\alpha} F\left(\left(x_{1}, \ldots, x_{n}\right), g\left(\left(x_{1}, \ldots, x_{n}\right)\right)\right)}{\partial^{\alpha} / \partial y^{\alpha} F\left(\left(x_{1}, \ldots, x_{n}\right), g\left(\left(x_{1}, \ldots, x_{n}\right)\right)\right) \cdot g\left(\left(x_{1}, \ldots, x_{n}\right)\right)^{\alpha-1}} .
$$

Proof. WLOG, $X$ is assumed to be an OB, represented by $B\left(\left(a_{1}, \ldots, a_{n}, b\right), \varepsilon_{0}\right)$. Let $\rho \in\left(0, \varepsilon_{0}\right)$. If we call $\delta=\sqrt{\varepsilon_{0}^{2}-\rho^{2}}$, it is verified that

$$
\left[\left\|\left(x_{1}, \ldots, x_{n}\right)-\left(a_{1}, \ldots, a_{n}\right)\right\|<\delta \text { and }|y-b|<\rho\right] \Rightarrow\left(x_{1}, \ldots, x_{n}, y\right) \in B\left(\left(a_{1}, \ldots, a_{n}, b\right), \varepsilon_{0}\right) \text {. }
$$

$$
\begin{aligned}
& F\left(a_{1}, \ldots, a_{n}, b-\rho\right)>0, \\
& F\left(a_{1}, \ldots, a_{n}, b-\rho\right)<0 .
\end{aligned}
$$
$\left(a_{1}, \ldots, a_{n}, y\right) \in B\left(\left(a_{1}, \ldots, a_{n}, b\right), \varepsilon_{0}\right)$.

Since $\partial^{\alpha} / \partial y^{\alpha} F\left(a_{1}, \ldots, a_{n}, b\right) \neq 0$, it is assumed to be positive (otherwise, $-F$ is considered instead of $F$ ). From fact that $F\left(a_{1}, \ldots, a_{n}, b\right)=0$, it follows that
By the continuity of $F$ at $\left(a_{1}, \ldots, a_{n}, b-\rho\right)$ and $\left(a_{1}, \ldots, a_{n}, b+\rho\right)$, there exists $\delta^{\prime} \in(0, \delta)$, such that

$$
\left\|\left(x_{1}, \ldots, x_{n}\right)-\left(a_{1}, \ldots, a_{n}\right)\right\|<\delta^{\prime} \Rightarrow\left[F\left(x_{1}, \ldots, x_{n}, b-\rho\right)>0 \text { and } F\left(x_{1}, \ldots, x_{n}, b+\rho\right)<0\right] .
$$

Since the function $y \mapsto F\left(x_{1}, \ldots, x_{n}, y\right)$ is CF on the interval $[b-\rho, b+\rho], \forall\left(x_{1}, \ldots, x_{n}\right) \in B\left(\left(a_{1}, \ldots, a_{n}\right), \delta^{\prime}\right)$, and via the classical Bolzano's theorem (BThm), it implies that $\exists y_{x} \in(b-\rho, b+\rho) \quad \ni F\left(x_{1}, \ldots, x_{n}, y_{x}\right)=0$, for each $x=\left(x_{1}, \ldots, x_{n}\right)$. Then, $y$ 's value is unique, since a function, whose derivative is positive, has more than zero. On the other hand, by having $U=B\left(\left(a_{1}, \ldots, a_{n}\right), \delta^{\prime}\right)$, for each $\left(x_{1}, \ldots, x_{n}\right) \in U$, there exists a unique $y$ $\ni F\left(x_{1}, \ldots, x_{n}, y\right)=0$, we can write $y=g\left(x_{1}, \ldots, x_{n}\right)$, and then, this function will be proven to be CF on $B\left(\left(a_{1}, \ldots, a_{n}\right), \delta^{\prime}\right)$. The continuity of the function $g$ at the point $\left(a_{1}, \ldots, a_{n}\right)$ is clear since for each $\rho>0, \exists$ a value $\delta^{\prime}>0 \ni$ : 


$$
\begin{aligned}
& \left\|\left(x_{1}, \ldots, x_{n}\right)-\left(a_{1}, \ldots, a_{n}\right)\right\|<\delta^{\prime} \Rightarrow\left|b-y_{x}\right| \\
& <\rho \Leftrightarrow\left|b-g\left(x_{1}, \ldots, x_{n}\right)\right|<\rho .
\end{aligned}
$$

The function $g$ continuity will be proven at any point $\left(x_{1}, \ldots, x_{n}\right) \in B\left(\left(a_{1}, \ldots, a_{n}\right), \delta^{\prime}\right)$ by simply substituting $B\left(\left(a_{1}, \ldots, a_{n}\right), \delta^{\prime}\right)$ for an OB: $B\left(\left(x_{1}, \ldots, x\right)\right)$ is contained in $B\left(\left(a_{1}, \ldots, a_{n}\right), \delta^{\prime}\right)$.

Finally, let us now show formula (35). By applying Theorem 14 to equation,

$$
\begin{aligned}
& F\left(x_{1}, \ldots, x_{n}, y\right)=0 \text {, we have } \\
& \frac{\partial^{\alpha}}{\partial x_{i}^{\alpha}} F(\mathbf{x}, g(\mathbf{x}))+\frac{\partial^{\alpha}}{\partial y^{\alpha}} F(\mathbf{x}, g(\mathbf{x})) \cdot g(\mathbf{x})^{\alpha-1} \cdot \frac{\partial^{\alpha}}{\partial x_{i}^{\alpha}} g(\mathbf{x})=0 .
\end{aligned}
$$

$\forall i=1,2, \ldots, n, \ni \mathbf{x}=\left(x_{1}, \ldots, x_{n}\right)$. Solving $\partial^{\alpha} / \partial x_{i}^{\alpha} g(\mathbf{x})$, we get (35). In addition, the formula (35) on the right side is continuous, so the continuity of $\mathrm{CoV}$ of PDrs $\partial^{\alpha} / \partial x_{i}^{\alpha} g(\mathbf{x}) \forall$, $i=1,2, \ldots, n$, follows.

Theorem 15 will provide us a help in computing CoV of PDrs of implicit function of SVs.

\section{Example 2. Consider}

$$
F(x, y, z)=x^{3}+3 y^{2}+4 x z^{2}-3 y z^{2}-5=0 .
$$

This equation's one solution is $(1,1,1) . F$ is obviously in $C_{\alpha}$ which is $\mathrm{OB}$, represented by $B\left((1,1,1), \varepsilon_{0}\right)$, with $x, y, z>0$, for some $\alpha \in(0,1]$, since

$$
\left.\frac{\partial^{\alpha}}{\partial z^{\alpha}} F(1,1,1)=\left(8 x z^{2-\alpha}-6 y z^{2-\alpha}\right)\right]_{(1,1,1)}=2 \neq 0 .
$$

Theorem 15 implies that there is a neighbourhood, $U \subset R^{2}$, of $(1,1) \ni \exists$ a UF: $z=g(x, y)$ satisfies the following: $g(1,1)=1$ and $F(x, y, g(x, y))=0, \forall(x, y) \in U$.

Moreover, $z=g(x, y)$ is $C_{\alpha}$ in $U$, and we have

$$
\begin{gathered}
\frac{\partial^{\alpha}}{\partial x^{\alpha}} g(x, y)=-\frac{\left(3 x+4 z^{2}\right) x^{1-\alpha}}{(8 x-6 y) z}, \\
\frac{\partial^{\alpha}}{\partial y^{\alpha}} g(x, y)=-\frac{\left(6 y-3 z^{2}\right) y^{1-\alpha}}{(8 x-6 y) z} .
\end{gathered}
$$

Finally, we obtain $\partial^{\alpha} / \partial x^{\alpha} g(1,1)=-(7 / 2)$ and $\partial^{\alpha} / \partial x^{\alpha} g(1,1)=-(3 / 2)$.

Finally, CoV of IFThm for a system of several equations and SRVs is obtained.

Theorem 16 (Conformable general implicit function theorem). Let $\alpha \in(0,1], \mathbf{F}: X \longrightarrow R^{m}$ be a VVF defined in an OS: $X \subset R^{n+m}, \ni \forall(\mathbf{x} ; \mathbf{y})=\left(x_{1}, \ldots, x_{n} ; y_{1}, \ldots, y_{m}\right) \in X$, each $x_{i}, y_{j}>0, \quad$ and point $(\mathbf{a} ; \mathbf{b})=\left(a_{1}, \ldots, a_{n} ; b_{1}, \ldots, b_{m}\right) \in X$. Assume that

(i) $\mathbf{F}(\mathbf{a} ; \mathbf{b})=0$

(ii) $\mathbf{F} \in C_{\alpha}\left(X, R^{m}\right)$ (iii) $\operatorname{det}\left[J_{y}^{\alpha} \mathbf{F}(\mathbf{a} ; \mathbf{b})\right] \neq 0$

Then, there is a neighbourhood, $U \subset R^{n}$, of a $\ni \exists$ a UF: $\mathbf{f}: U \longrightarrow R^{m}, \mathbf{x} \mapsto \mathbf{y}=\mathbf{f}(\mathbf{x})$ that satisfies $\mathbf{f}(\mathbf{a})=\mathbf{b}$ and $\mathbf{F}(\mathbf{x} ; \mathbf{f}(\mathbf{x}))=0, \forall \mathbf{x} \in U$.

Finally, $\mathbf{y}=\mathbf{f}(\mathbf{x})$ is class $C_{\alpha}$ in $U$, and for every $i=1,2, \ldots, n$, we have

$$
\left[\frac{\partial^{\alpha} \mathbf{f}}{\partial x_{i}^{\alpha}}\right]^{t}=-\left(\mathbf{f}^{\alpha-1}\right)^{-1} \cdot\left(J_{y}^{\alpha} \mathbf{F}\right)^{-1} \cdot\left[\frac{\partial^{\alpha} \mathbf{F}}{\partial x_{i}^{\alpha}}\right]^{t},
$$

where

$$
\begin{aligned}
& {\left[\frac{\partial^{\alpha} \mathbf{f}}{\partial x_{i}^{\alpha}}\right]=\left(\frac{\partial^{\alpha} f_{1}}{\partial x_{i}^{\alpha}}, \ldots, \frac{\partial^{\alpha} f_{m}}{\partial x_{i}^{\alpha}}\right),} \\
& \mathbf{f}^{\alpha-1}=\left(\begin{array}{ccc}
f_{1}^{\alpha-1} & \ldots & 0 \\
\vdots & \ldots & \vdots \\
0 & \ldots & f_{m}^{\alpha-1}
\end{array}\right), \\
& J_{y}^{\alpha} \mathbf{F}=\left(\begin{array}{ccc}
\frac{\partial^{\alpha} F_{1}}{\partial y_{1}^{\alpha}} & \cdots & \frac{\partial^{\alpha} F_{1}}{\partial y_{m}^{\alpha}} \\
\cdots & \cdots & \cdots \\
\frac{\partial^{\alpha} F_{m}}{\partial y_{1}^{\alpha}} & \cdots & \frac{\partial^{\alpha} F_{m}}{\partial y_{m}^{\alpha}}
\end{array}\right) \\
& {\left[\frac{\partial^{\alpha} \mathbf{F}}{\partial x_{i}^{\alpha}}\right]=\left(\frac{\partial^{\alpha} F_{1}}{\partial x_{i}^{\alpha}}, \ldots, \frac{\partial^{\alpha} F_{m}}{\partial x_{i}^{\alpha}}\right) .}
\end{aligned}
$$

Proof. The proof of existence and uniqueness of the implicit function is done similar to the traditional multivariable calculus by applying mathematical induction on $q$ and using the conformable implicit function theorem for several variables [28].

Let us now show formula (44). Assume that a system with several equations and SRVs is expressed as

$$
F(\mathbf{x} ; \mathbf{y})=0 \text { or }\left\{\begin{array}{c}
F_{1}\left(x_{1}, \ldots, x_{n} ; y_{1}, \ldots, y_{m}\right)=0 \\
\vdots \\
F_{m}\left(x_{1}, \ldots, x_{n} ; y_{1}, \ldots, y_{m}\right)=0
\end{array},\right.
$$

which satisfies hypotheses (i)-(iii) of the theorem; then, this system is defined in a neighbourhood, $U \subset R^{n}$, of $\mathbf{a}$, the implicit function $\mathbf{y}=\mathbf{f}(\mathbf{x})$ class $C^{\alpha}$ in $U$, such that $\mathbf{f}(\mathbf{a})=\mathbf{b}$ which satisfies equation (1), i.e.,

$$
F(\mathbf{x} ; \mathbf{f}(\mathbf{x}))=0 \text { or }\left\{\begin{array}{c}
F_{1}\left(\mathbf{x} ; f_{1}(\mathbf{x}), \ldots, f_{m}(\mathbf{x})\right)=0 \\
\ldots \\
F_{m}\left(\mathbf{x} ; f_{1}(\mathbf{x}), \ldots, f_{m}(\mathbf{x})\right)=0
\end{array}\right.
$$

By applying CoV of CR to the above equation, we have 


$$
\left.\begin{array}{c}
\frac{\partial^{\alpha} F_{1}}{\partial x_{i}^{\alpha}}+\frac{\partial^{\alpha} F_{1}}{\partial y_{1}^{\alpha}} \cdot f_{1}^{\alpha-1} \cdot \frac{\partial^{\alpha} f_{1}}{\partial x_{i}^{\alpha}}+\cdots+\frac{\partial^{\alpha} F_{1}}{\partial y_{m}^{\alpha}} \cdot f_{m}^{\alpha-1} \cdot \frac{\partial^{\alpha} f_{m}}{\partial x_{i}^{\alpha}}=0 \\
\vdots \\
\frac{\partial^{\alpha} F_{m}}{\partial x_{i}^{\alpha}}+\frac{\partial^{\alpha} F_{m}}{\partial y_{1}^{\alpha}} \cdot f_{1}^{\alpha-1} \cdot \frac{\partial^{\alpha} f_{1}}{\partial x_{i}^{\alpha}}+\cdots+\frac{\partial^{\alpha} F_{m}}{\partial y_{m}^{\alpha}} \cdot f_{m}^{\alpha-1} \cdot \frac{\partial^{\alpha} f_{m}}{\partial x_{i}^{\alpha}}=0
\end{array}\right\}
$$

for all $i=1,2, \ldots, n$.

In addition, the MF of equation (48) is given as follows:

$$
\begin{aligned}
& \left(\begin{array}{c}
\frac{\partial^{\alpha} F_{1}}{\partial x_{i}^{\alpha}} \\
\vdots \\
\frac{\partial^{\alpha} F_{m}}{\partial x_{i}^{\alpha}}
\end{array}\right)=-\left(\begin{array}{ccc}
\frac{\partial^{\alpha} F_{1}}{\partial y_{1}^{\alpha}} & \ldots & \frac{\partial^{\alpha} F_{1}}{\partial y_{m}^{\alpha}} \\
\ldots & \ldots & \ldots \\
\frac{\partial^{\alpha} F_{m}}{\partial y_{1}^{\alpha}} & \ldots & \frac{\partial^{\alpha} F_{m}}{\partial y_{m}^{\alpha}}
\end{array}\right) \cdot\left(\begin{array}{ccc}
f_{1}^{\alpha-1} & \ldots & 0 \\
\vdots & \ldots & \vdots \\
0 & \ldots & f_{m}^{\alpha-1}
\end{array}\right) \cdot\left(\begin{array}{c}
\frac{\partial^{\alpha} f_{1}}{\partial x_{i}^{\alpha}} \\
\vdots \\
\frac{\partial^{\alpha} F_{m}}{\partial x_{i}^{\alpha}}
\end{array}\right) \\
& \text { or }\left[\frac{\partial^{\alpha} \mathbf{F}}{\partial x_{i}^{\alpha}}\right]^{t}=-J_{y}^{\alpha} \mathbf{F} \cdot \mathbf{f}^{\alpha-1} \cdot\left[\frac{\partial^{\alpha} \mathbf{f}}{\partial x_{i}^{\alpha}}\right]^{t} .
\end{aligned}
$$
have

Since $J_{y}^{\alpha} \mathbf{F}$ and $\mathbf{f}^{\alpha-1}$ are regular matrices by hypothesis, we

$$
\begin{aligned}
& \left(\begin{array}{c}
\frac{\partial^{\alpha} f_{1}}{\partial x_{i}^{\alpha}} \\
\vdots \\
\frac{\partial^{\alpha} F_{m}}{\partial x_{i}^{\alpha}}
\end{array}\right)^{-1}=-\left(\begin{array}{ccc}
f_{1}^{\alpha-1} & \ldots & 0 \\
\vdots & \ldots & \vdots \\
0 & \ldots & f_{m}^{\alpha-1}
\end{array}\right)^{-1} \cdot\left(\begin{array}{ccc}
\frac{\partial^{\alpha} F_{1}}{\partial y_{1}^{\alpha}} & \ldots & \frac{\partial^{\alpha} F_{1}}{\partial y_{m}^{\alpha}} \\
\ldots & \ldots & \ldots \\
\frac{\partial^{\alpha} F_{m}}{\partial y_{1}^{\alpha}} & \ldots & \frac{\partial^{\alpha} F_{m}}{\partial y_{m}^{\alpha}}
\end{array}\right)^{-1} \cdot\left(\begin{array}{c}
\frac{\partial^{\alpha} F_{1}}{\partial x_{i}^{\alpha}} \\
\vdots \\
\frac{\partial^{\alpha} F_{m}}{\partial x_{i}^{\alpha}}
\end{array}\right) \\
& \text { or }\left[\frac{\partial^{\alpha} \mathbf{f}}{\partial x_{i}^{\alpha}}\right]^{t}=-\left(\mathbf{f}^{\alpha-1}\right)^{-1} \cdot\left(J_{y}^{\alpha} \mathbf{F}\right)^{-1} \cdot\left[\frac{\partial^{\alpha} \mathbf{F}}{\partial x_{i}^{\alpha}}\right]^{t}
\end{aligned}
$$

which completes the proof.

We will now show that Theorem 16 can be used to compute $\mathrm{CoV}$ of PDrs of systems with several equations and SRVs.

Example 3. Consider a system of two equations and two real variables:

$$
\left\{\begin{array}{l}
F_{1}(x, y, z, w)=x^{2}+y^{2}+z^{2}+w^{2}-6=0 \\
F_{2}(x, y, z, w)=x^{2}-y^{2}+z^{2}-w^{2}=0
\end{array}\right.
$$

One solution of this equation is $(x, y, z, w)=(1,1, \sqrt{2}, \sqrt{2})$. Clearly, $\mathbf{F}=\left(F_{1}, F_{2}\right)$ is in $C_{\alpha}$, which is OB: $B\left((1,1, \sqrt{2}, \sqrt{2}), \varepsilon_{0}\right)$, with $x, y, z, w>0$, for some $\alpha \in(0,1]$, since 


$$
\operatorname{det}\left[J_{z, w}^{\alpha} \mathbf{F}((\sqrt{2}, \sqrt{2}, 1,1))\right]=\operatorname{det}\left[\left(\begin{array}{cc}
2 z^{2-\alpha} & 2 w^{2-\alpha} \\
2 z^{2-\alpha} & -2 w^{2-\alpha}
\end{array}\right)\right]_{(1,1, \sqrt{2}, \sqrt{2})}=-\frac{32}{2^{\alpha}} \neq 0
$$

Theorem 16 implies that there is a neighbourhood, $U \subset R^{2}$, of $(\sqrt{2}, \sqrt{2}) \ni \exists$ a UF: $\mathbf{f}=\left(f_{1}, f_{2}\right)$ given by

$$
\left\{\begin{array}{l}
z=f_{1}(x, y) \\
w=f_{2}(x, y)
\end{array}\right.
$$

$$
\begin{aligned}
& \left\{\begin{array}{l}
f_{1}(1,1)=\sqrt{2}, \\
f_{2}(1,1)=\sqrt{2},
\end{array}\right. \\
& \left\{\begin{array}{l}
F_{1}\left(x, y, f_{1}(x, y), f_{2}(x, y)\right)=0, \\
F_{2}\left(x, y, f_{1}(x, y), f_{2}(x, y)\right)=0,
\end{array} \quad \forall(x, y) \in U .\right.
\end{aligned}
$$

that satisfies

$$
\begin{aligned}
& \left(\begin{array}{l}
\frac{\partial^{\alpha} f_{1}}{\partial x^{\alpha}} \\
\frac{\partial^{\alpha} f_{2}}{\partial x^{\alpha}}
\end{array}\right)=-\left(\begin{array}{cc}
z^{\alpha-1} & 0 \\
0 & w^{\alpha-1}
\end{array}\right)^{-1} \cdot\left(\begin{array}{cc}
2 z^{2-\alpha} & 2 w^{2-\alpha} \\
2 z^{2-\alpha} & -2 w^{2-\alpha}
\end{array}\right)^{-1} \cdot\left(\begin{array}{c}
2 x^{2-\alpha} \\
2 x^{2-\alpha}
\end{array}\right)=-\frac{1}{4} \cdot\left(\begin{array}{cc}
z^{1-\alpha} & 0 \\
0 & w^{1-\alpha}
\end{array}\right) \cdot\left(\begin{array}{cc}
z^{\alpha-2} & z^{\alpha-2} \\
w^{\alpha-2} & -w^{\alpha-2}
\end{array}\right) \\
& \left(\begin{array}{c}
2 x^{2-\alpha} \\
2 x^{2-\alpha}
\end{array}\right)=-\frac{1}{4} \cdot\left(\begin{array}{cc}
z^{-1} & z^{-1} \\
w^{-1} & -w^{-1}
\end{array}\right) \cdot\left(\begin{array}{c}
2 x^{2-\alpha} \\
2 x^{2-\alpha}
\end{array}\right)=\left(\begin{array}{c}
-\frac{x^{2-\alpha}}{z} \\
0
\end{array}\right) \\
& \left(\begin{array}{c}
\frac{\partial^{\alpha} f_{1}}{\partial y^{\alpha}} \\
\frac{\partial^{\alpha} f_{2}}{\partial y^{\alpha}}
\end{array}\right)=-\left(\begin{array}{cc}
z^{\alpha-1} & 0 \\
0 & w^{\alpha-1}
\end{array}\right)^{-1} \cdot\left(\begin{array}{cc}
2 z^{2-\alpha} & 2 w^{2-\alpha} \\
2 z^{2-\alpha} & -2 w^{2-\alpha}
\end{array}\right)^{-1} \cdot\left(\begin{array}{c}
2 y^{2-\alpha} \\
-2 y^{2-\alpha}
\end{array}\right)=-\frac{1}{4} \cdot\left(\begin{array}{cc}
z^{1-\alpha} & 0 \\
0 & w^{1-\alpha}
\end{array}\right) \cdot\left(\begin{array}{cc}
z^{\alpha-2} & z^{\alpha-2} \\
w^{\alpha-2} & -w^{\alpha-2}
\end{array}\right) \\
& \cdot\left(\begin{array}{c}
2 y^{2-\alpha} \\
-2 y^{2-\alpha}
\end{array}\right)=-\frac{1}{4} \cdot\left(\begin{array}{cc}
z^{-1} & z^{-1} \\
w^{-1} & -w^{-1}
\end{array}\right) \cdot\left(\begin{array}{c}
2 y^{2-\alpha} \\
-2 y^{2-\alpha}
\end{array}\right)=\left(\begin{array}{c}
0 \\
-\frac{y^{2-\alpha}}{w}
\end{array}\right) .
\end{aligned}
$$

Finally, we have

$$
\begin{aligned}
& \left(\begin{array}{l}
\frac{\partial^{\alpha} f_{1}}{\partial x^{\alpha}} \\
\frac{\partial^{\alpha} f_{2}}{\partial x^{\alpha}}
\end{array}\right)_{(1,1, \sqrt{2}, \sqrt{2})}=\left(\begin{array}{c}
-\frac{1}{\sqrt{2}} \\
0
\end{array}\right) \\
& \left(\begin{array}{l}
\frac{\partial^{\alpha} f_{1}}{\partial y^{\alpha}} \\
\frac{\partial^{\alpha} f_{2}}{\partial y^{\alpha}}
\end{array}\right)_{(1,1, \sqrt{2}, \sqrt{2})}=\left(\begin{array}{c}
0 \\
1 \\
-\frac{1}{\sqrt{2}}
\end{array}\right) .
\end{aligned}
$$

According to all of our numerical examples in this work, with the help of our investigation to all proposed theorems and properties in this article, the numerical examples show that all our obtained results are valid and efficient. As seen in all these examples, the computations are simple and cost- efficient, which are important when scientific phenomena are modelled in the sense of conformable calculus. All in all, the simplicity of computations is always needed in modeling applications in comparison to other complicated computations that are needed using other approaches in other fractional operators. All our obtained results in this work are accurate because our results coincide with the usual integerorder results.

\section{Conclusion}

A new investigation on the $\mathrm{CoV}$ of multivariable calculus has been discussed in this work in detail. The $\alpha$-derivative of a function of SVs and all related properties have been investigated. The CoV of CR for functions of SVs has also been studied. The CoV of IFThm has been presented in the last part of our results, and numerical experiments have been conducted to support our theoretical results. The findings of this investigations show that all results formulated via CoD 
coincide with the ones in the traditional integer case. All results in this work can be potentially applied in modeling oceanographic phenomena such as Stommel's box model of thermohaline circulation and other related models where this study's analysis can be further extended or generalized in future works for various related physical models.

\section{Data Availability}

No data were used to support this study.

\section{Conflicts of Interest}

All authors declare that they have no conflicts of interest.

\section{Authors' Contributions}

Mohammed K. A. Kaabar involved in actualization, developed methodology, performed formal analysis, validated and investigated the study, prepared the initial draft, and supervised and edited the original draft. Francisco Martínez, Inmaculada Martínez, and Silvestre Paredes involved in actualization, developed methodology, performed formal analysis, validated and investigated the study, and prepared the initial draft. Zailan Siri developed methodology, performed formal analysis, validated and investigated the study, and prepared the initial draft. All authors read and approved the final version.

\section{References}

[1] M. M. A. Khater, M. S. Mohamed, and R. A. M. Attia, "On semi analytical and numerical simulations for a mathematical biological model; the time-fractional nonlinear Kolmogorov-Petrovskii-Piskunov (KPP) equation," Chaos, Solitons \& Fractals, vol. 144, Article ID 110676, 2021.

[2] M. A. Khater, K. S. Nisar, and M. S. Mohamed, "Numerical investigation for the fractional nonlinear space-time telegraph equation via the trigonometric Quintic B-spline scheme," Mathematical Methods in the Applied Sciences, vol. 44, no. 6, pp. 4598-4606, 2021.

[3] M. M. A. Khater, M. S. Mohamed, and S. K. Elagan, "Diverse accurate computational solutions of the nonlinear Klein-Fock-Gordon equation," Results in Physics, vol. 23, Article ID 104003, 2021.

[4] M. F. Li, J. R. Ren, and T. Zhu, "Fractional vector calculus and fractional special function," arXiv preprint:1001.2889, 2010.

[5] D. J. Wollkind and B. J. Dichone, Comprehensive Applied Mathematical Modeling in the Natural and Engineering Sciences, Springer International Publishing, Berlin, Germany, 2017.

[6] P. J. Olver, Introduction to Partial Differential Equations, Springer International Publishing, Berlin, Germany, 2014.

[7] J. D. Krehbiel and J. B. Freund, "Stokes flow inside a sphere in an inviscid extensional flow," Zeitschrift für Angewandte Mathematik und Physik, vol. 68, no. 4, pp. 68-81, 2017.

[8] A. A. Kilbas, H. M. Srivastava, and J. J. Trujillo, "Theory and applications of fractional differential equations," in NorthHolland Mathematics StudiesVol. 204, Elsevier, Amsterdam, Netherlands, 2006.

[9] K. S. Miller, An Introduction to Fractional Calculus and Fractional Differential Equations, John Wiley and Sons, Hoboken, NJ, USA, 1993.
[10] R. Khalil, M. Al Horani, A. Yousef, and M. Sababheh, "A new definition of fractional derivative," Journal of Computational and Applied Mathematics, vol. 264, pp. 65-70, 2014.

[11] R. Khalil, M. Al Horani, and M. Abu Hammad, "Geometric meaning of conformable derivative via fractional cords," The Journal of Mathematics and Computer Science, vol. 19, no. 4, pp. 241-245, 2019.

[12] T. Abdeljawad, "On conformable fractional calculus," Journal of Computational and Applied Mathematics, vol. 279, pp. 57-66, 2015.

[13] O. S. Iyiola and E. R. Nwaeze, "Some new results on the new conformable fractional calculus with application using D'Alambert approach," Progress in Fractional Differentiation and Applications, vol. 2, no. 2, pp. 115-122, 2016.

[14] A. Atangana, D. Baleanu, and A. Alsaedi, "New properties of conformable derivative," Open Mathematics, vol. 13, pp. 5763, 2015.

[15] N. Yazici and U. Gözütok, "Multivariable conformable fractional calculus," Filomat, vol. 32, no. 2, pp. 45-53, 2018.

[16] F. Martínez, I. Martínez, and S. Paredes, "Conformable Euler's theorem on homogeneous functions," Computational Mathematical Methods, vol. 1, no. 5, pp. 1-11, 2018.

[17] A. A. Hyder and A. H. Soliman, "Exact solutions of space-time local fractal nonlinear evolution equations: a generalized conformable derivative approach," Results in Physics, vol. 17, Article ID 103135, 2020.

[18] A. A. Hyder, "White noise theory and general improved Kudryashov method for stochastic nonlinear evolution equations with conformable derivatives," Advances in Difference Equations, vol. 202019 pages, 2020.

[19] A. H. Soliman and A. A. Hyder, "Closed-form solutions of stochastic $\mathrm{KdV}$ equation with generalized conformable derivatives," Physica Scripta, vol. 95, no. 6, Article ID 065219, 2020.

[20] V. E. Tarasov, "No violation of the Leibniz rule. No fractional derivative," Communications in Nonlinear Science and Numerical Simulation, vol. 18, no. 11, pp. 2945-2948, 2013.

[21] R. Khalil, M. Al Horani, A. Yousef, and M. Sababheh, "Fractional analytic functions," Far East Journal of Mathematical Sciences, vol. 103, no. 1, pp. 113-123, 2018.

[22] S. Uçar and N. Y. Ózgür, "Complex conformable derivative," Arabian Journal of Geosciences, vol. 12, no. 201, 2019.

[23] F. Martínez, I. Martínez, I. Martínez, M. K. A. Kaabar, and S. Paredes, "New results on complex conformable integral," AIMS Mathematics, vol. 5, no. 6, pp. 7695-7710, 2020.

[24] H. Kiskinov, M. Petkova, A. Zahariev, and M. Veselinova, "Some results about conformable derivatives in Banach spaces and an application to the partial differential equations," in AIP Conference Proceedingsvol. 2333, no. 1, AIP Publishing LLC, Article ID 120002, 2021.

[25] U. Gözütok, H. A. Çoban, and Y. Sağıroğlu, "Frenet frame with respect to conformable derivative," Filomat, vol. 33, no. 6, pp. 1541-1550, 2019.

[26] J. C. Mayo-Maldonado, G. Fernandez-Anaya, and O. F. RuizMartinez, "Stability of conformable linear differential systems: a behavioural framework with applications in fractional-order control," IET Control Theory \& Applications, vol. 14, no. 18, pp. 2900-2913, 2020.

[27] M. Al Horani and R. Khalil, "Total fractional differential with applications to exact fractional differential equations," International Journal of Computer Mathematics, vol. 95, no. 6-7, pp. 1444-1452, 2018.

[28] J. E. Marsden and M. J. Hoffman, Vector Calculus, W. H. Freeman and Company, New York, NY, USA, 4th edition, 1996. 